\title{
Reporte y distribución potencial de una palma exótica ornamental (Roystonea oleracea) en Ecuador
}

New record and potential distribution of an exotic ornamental palm

(Roystonea oleracea) in Ecuador

\author{
Ileana Herrera ${ }^{1,6}$, José I. Hernández-Rosas ${ }^{2,3}$, Cesar F. Suárez ${ }^{2}$, Xavier Cornejo $^{4}$, Alix Amaya ${ }^{2}$, \\ Estefany Goncalves ${ }^{5} \&$ Claudia Ayala ${ }^{2}$
}

\begin{abstract}
Resumen
La palma Roystonea oleracea, nativa del Caribe, ha sido introducida en varios países tropicales como planta ornamental e invade en humedales de Brasil, Guyana y Panamá. En este estudio reportamos la presencia de $R$. oleracea en la región de la costa del Ecuador donde es ampliamente cultivada como ornamental. Mediante una imagen de satélite estimamos la abundancia y cobertura de la especie en Isla Santay, un humedal Ramsar ubicado en el río Guayas cerca del perímetro urbano al sur de la ciudad de Guayaquil. También, realizamos un modelo de distribución potencial de R. oleracea en Ecuador, usando BIOCLIM. En Isla Santay, la especie está naturalizada y cubre alrededor de 43 hectáreas. El modelo de distribución potencial muestra que, además de Isla Santay, hay otros cinco humedales Ramsar en la costa ecuatoriana susceptibles climáticamente al establecimiento de $R$. oleracea. Debido al potencial invasivo de esta especie en humedales, se recomienda prohibir su uso como planta ornamental en áreas cercanas a humedales en Ecuador. Estudios que monitoreen la naturalización e impactos ecológicos de $R$. oleracea en humedales de Ecuador son requeridos para establecer prioridades de manejo y contribuir con la conservación de estos importantes ecosistemas.

Palabras clave: Arecaceae; distribución potencial; especies introducidas; humedales Ramsar; invasión de plantas.
\end{abstract}

\begin{abstract}
The Roystonea oleracea palm, a native of the Caribbean, has been introduced in some tropical countries as an ornamental plant and it invades the wetlands of Brazil, Guyana and Panama. In this study we report the presence of $R$. oleracea in the coastal region of Ecuador where it is widely cultivated as an ornamental. Using a satellite image, we estimated the abundance and coverage of this species at Isla Santay, a Ramsar wetland. We also made a potential distribution model of $R$. oleracea in Ecuador, using BIOCLIM. At Isla Santay, this species has been naturalized and covers about 43 hectares. The potential distribution model shows that, in addition to Isla Santay, there are five other Ramsar wetlands on the coast of Ecuador that are susceptible climatically to establishment of $R$. oleracea. Due to the invasive potential of this species in wetlands we recommend that its use as an ornamental plant be prohibited in areas near wetlands in Ecuador. Studies must be carried out to monitor naturalization and ecological impacts of $R$. oleracea in Ecuadorian wetlands in order to establish management priorities and contribute to the conservation of these important ecosystems. Key words: Arecaceae; potential distribution; introduced species, Ramsar wetlands; plant invasion.
\end{abstract}

\footnotetext{
${ }^{1}$ Universidad Espíritu Santo, Escuela de Ciencias Ambientales, Km 2,5 vía la Puntilla, 091650, Samborondón, Ecuador.

${ }^{2}$ Universidad Agraria del Ecuador, Facultad de Ciencias Agrarias, Av. 25 de julio, 090104, Guayaquil, Ecuador.

${ }^{3}$ Universidad Central de Venezuela, Facultad de Ciencias, Escuela de Biología, Los Chaguaramos, 1010-A, Caracas, Venezuela.

${ }^{4}$ Universidad de Guayaquil, Facultad de Ciencias Naturales, Herbario GUAY, Av. Juan Tanca Marengo s.n., y Las Aguas, P.O. Box 09-01-10634, Guayaquil, Ecuador.

${ }^{5}$ Universidad de Chile, Facultad de Ciencias, Depto. Ciencias Ecológicas, Lab. Ecología Terrestre, Las Palmeras 3425 Ñuñoa, Santiago, 7800003, Santiago, Chile.

${ }^{6}$ Autor para la correspondencia: herrera.ita@gmail.com
} 


\section{Introducción}

La invasión por especies de plantas introducidas modifica la estructura y función de los ecosistemas al reducir la diversidad de especies nativas y modificar la actividad microbiana y reservorios de nutrientes en el suelo (Vilà et al. 2011). El proceso de invasión por especies vegetales ocurre en tres fases (Shigesada \& Kawasaki 1997; Mack et al. 2000). La primera es la fase de introducción, que puede ser accidental o deliberada, e involucra el transporte de especies, mediado por actividades humanas, hacia áreas bio-geográficamente aisladas del área de distribución natural de la especie transportada (denominada especie exótica). La segunda fase es la de establecimiento, durante la cual la especie (denominada especie naturalizada) es capaz de reproducirse sin intervención del hombre en ecosistemas naturales. La última fase del proceso de invasión es la de expansión, que se alcanza cuando la especie aumenta exponencialmente su área de distribución, en esta fase la especie es categorizada como una especie invasora (Shigesada \& Kawasaki 1997; Mack et al. 2000).

Aunque todos los ecosistemas son vulnerables a la invasión por plantas, la alta vulnerabilidad de las islas oceánicas a las invasiones biológicas es ampliamente reconocida. Algunos autores lo atribuyen a que las islas oceánicas tienen baja riqueza y poblaciones pequeñas de especies nativas (Rejmánek 1996; Elton 2000). Otros autores sugieren que esta vulnerabilidad puede deberse a que las especies nativas de las islas oceánicas muestran alto grado de especiación y endemismo, lo que se refleja en estructuras tróficas simples y en la baja capacidad competitiva de las especies, en comparación con las especies de los continentes (D’Antonio \& Dudley 1995; Lonsdale 1999). Poco se ha discutido sobre la vulnerabilidad a las invasiones biológicas de las islas continentales con respecto a las oceánicas. Una diferencia obvia es que las islas continentales no se encuentran totalmente aisladas del continente. Sin embargo, las islas continentales pueden compartir con las oceánicas la baja riqueza de especies y el bajo tamaño poblacional de sus especies nativas como respuesta al área geográfica limitada que caracteriza a las islas (p.e., Kallimanis et al. 2010). Estas características pueden incrementar la vulnerabilidad de los ecosistemas de las islas continentales a las invasiones biológicas.
Muchas introducciones de especies vegetales son deliberadas (p.e., Reichard 1997; Groves 1998), estas introducciones se caracterizan por ocurrir en múltiples eventos y por el movimiento de un gran número de individuos (Wilson et al. 2009). En algunas regiones, la mayor proporción de las especies vegetales son introducidas deliberadamente por el hombre debido a su valor ornamental (Groves 1998; Mack \& Erneberg 2002). Dentro de estas especies, las palmas son uno de los grupos más valorados (Maciel \& Mogollón 1995). En la Guayana Francesa, por ejemplo, cerca del $20 \%$ de las especies introducidas son palmas (96 especies; Delnatte \& Meyer 2012) y en islas tropicales varias especies introducidas de palmas son ahora reportadas como naturalizadas o invasoras (Meyer et al. 2007). En general, las poblaciones de especies de palmas tienden a presentar una distribución agregada, lo que podría favorecer el establecimiento de poblaciones densas, característica que podría propiciar que algunas de estas especies se conviertan en invasoras. De hecho, a escala global se han reportado 35 especies de palmas invasoras (<http://www.issg.org $>)$. Un caso bien documentado en Sudamérica es la invasión por la palma australiana Archontophoenix cunninghamiana en parches remanentes de la Mata Atlántica en Brasil (Dislich et al. 2002).

En Ecuador continental, la palma real o chaguaramo (Roystonea oleracea, Arecaceae) ha sido introducida deliberadamente como planta ornamental a lo largo de toda la región de la costa. Además de ser cultivada, $R$. oleracea mantiene poblaciones autosustentables en el humedal Ramsar "Isla Santay" (X. Cornejo, observación personal), una isla continental ubicada en la costa ecuatoriana. Sin embargo, esta especie no ha sido registrada en el Catálogo de las plantas vasculares del Ecuador publicado por Jørgensen \& León-Yánez (1999).

Roystonea oleracea es nativa de la región del Caribe y ha sido introducida en muchos países tropicales (Kahn \& Moussa 1997). Reportes previos catalogan a $R$. oleracea como una especie invasora o naturalizada en humedales en Brasil, Guyana y Panamá (Henderson et al. 1995; Svenning 2002; Nascimento et al. 2013). En este trabajo nos propusimos: 1) reportar la presencia de $R$. oleracea en Ecuador; 2) estimar la abundancia en Isla Santay y la distribución potencial en Ecuador de $R$. oleracea; y 3 ) examinar la susceptibilidad climática de otros humedales Ramsar del Ecuador 
al establecimiento de esta palma introducida. También, investigamos sobre los posibles riesgos asociados a la introducción de esta especie en áreas susceptibles e hicimos inferencias sobre posibles estrategias para manejar las poblaciones en Isla Santay. Nuestra investigación representa uno de los escasos estudios publicados sobre especies de plantas introducidas en Ecuador continental. Además, de reportar la presencia de una especie de palma naturalizada que podría tener el potencial de invadir, nuestro estudio contribuirá a detectar humedales vulnerables a la invasión por esta especie en Ecuador.

\section{Materiales y Métodos}

En visitas exploratorias a lo largo de la región de la costa del Ecuador se detectó la presencia de Roystonea oleracea. En Isla Santay, se detectaron varias poblaciones establecidas. Isla Santay se encuentra ubicada en el río Guayas (cerca de la ciudad de Guayaquil) en la región de la Costa del Ecuador (2¹3'S, 7950'E) tiene una superficie de 2.179 hectáreas, y es catalogada como un humedal Ramsar y un área protegida del Ecuador (<http://www.ramsar.org/es/isla-santay-0>). La temperatura promedio anual es de $35^{\circ} \mathrm{C}$ y la precipitación promedio total es de $1.113 \mathrm{~mm}$ (Navarrete 2008). La isla alberga varios tipos de vegetación tales como bosques secos, bosques inundables, manglares y humedales (Navarrete 2008). Una de las amenazas que podría afectar el estatus de protección de Isla Santay es la alta influencia de turistas (Navarrete 2008).

En Isla Santay realizamos observaciones in situ de los aspectos ecológicos y botánicos de individuos $(n=10)$ en poblaciones de la especie y se colectaron e identificaron individuos adultos en varios sectores de la Isla. Con base en estas observaciones y revisiones bibliográficas, se realizó una descripción detallada de la especie.

Abundancia, cobertura y densidad de Roystonea oleracea en Isla Santay

Para estimar la abundancia y cobertura de individuos adultos de $R$. oleracea en Isla Santay cuantificamos el número de copas observadas en una imagen de satélite (resolución de 0.56 $\mathrm{m}^{2}$, año: 2014). Usando ArcGIS (ver. 10.1), la imagen satelital se dividió en cuadrantes de 0.25 $\mathrm{Km}^{2}$, con el fin de sectorizar el área y facilitar la cuantificación de las copas de $R$. oleracea. Para estimar la cobertura promedio de la copa de una palma, medimos sobre la imagen satelital el área de la copa de 25 individuos aislados seleccionados aleatoriamente. El radio promedio de una copa fue utilizado como referencia para realizar un buffer sobre cada punto de presencia de un individuo adulto de $R$. oleracea. Luego, se estimó el área delimitada por el buffer para estimar la cobertura de las copas de $R$. oleracea en Isla Santay. Para identificar los parches más densos, realizamos un análisis de la densidad de palmas por hectárea usando la función de Kernel en ArcGis (ver. 10.1).

Distribución potencial de Roystonea oleracea en Ecuador

Para obtener la distribución potencial de $R$. oleracea en el Ecuador realizamos un modelo de distribución potencial utilizando el algoritmo de BIOCLIM en el programa Diva-Gis $(<\mathrm{http} / / /$ www.diva-gis.org/>). BIOCLIM determina el conjunto de atributos climáticos asociados a los registros de presencia y, así con base en los límites observados de cada variable deriva un perfil de hábitat o clima adecuado para la especie (Carpenter et al. 1993). Una vez definidas las áreas (pixeles) con condiciones climáticas similares, BIOCLIM genera mapas que muestran la distribución potencial de la especie basado en variables climáticas y registros geo-referenciados de la especie. Esta distribución potencial evidencia las áreas susceptibles al establecimiento de $R$. oleracea en el Ecuador. Para la construcción de los modelos utilizamos cuatro variables climáticas: i) Temperatura media anual (Bio1), ii) Rango de temperatura diario promedio (Bio2), iii) Precipitación anual (Bio12) y iv) Estacionalidad de la precipitación (i.e., coeficiente de variación de la precipitación, Bio15). Estas variables climáticas se obtuvieron desde la base de datos climática WorldCLIM (Hijmans et al. 2005; disponibles en: <http://www.worldclim.org/download $>$ ). Para seleccionar las variables climáticas y evitar un sobre-ajuste del modelo, realizamos un análisis de correlación para escoger sólo aquellas variables con baja correlación entre sí $(<0.7)$. Para obtener una muestra lo más representativa posible de las condiciones climáticas que puede abarcar la especie, se obtuvieron registros globales de presencia de la especie a través del portal "Red de Información de Biodiversidad Global" (Global Biodiversity Information Facility - GBIF, <http:// www.gbif.org/>). Se seleccionaron únicamente los registros $(n=19)$ de presencia con una fecha igual o posterior a 1950, debido a que las variables 
climáticas disponibles para los análisis fueron generadas con datos climáticos posteriores a esa fecha. El modelo se evaluó al calcular la tasa de omisión, es decir, la cantidad de registros de presencia que se encuentran en áreas predichas como no adecuadas por el modelo. Esto permite determinar qué tan acertada es la calibración del modelo obtenido. En este caso la tasa de omisión fue menor al $1 \%$. Con la finalidad de identificar los humedales del Ecuador que podrían ser susceptibles climáticamente al establecimiento de $R$. oleracea, las coordenadas de los humedales Ramsar del Ecuador ( <http://www.ramsar.org/es/ countries/ecuador $>$ ) fueron sobrepuestas sobre el modelo de distribución potencial de $R$. oleracea.

Para describir las condiciones climáticas bajo las cuales la especie podría invadir en Ecuador (condiciones más susceptibles a la invasión), se estimó el valor de las variables climáticas en cada pixel predicho por el modelo con alta y muy alta susceptibilidad (áreas de color naranja y rojo). Este valor se obtuvo en Arcgis 9.3 y se graficaron en un histograma de frecuencias los valores obtenidos para cada variable.

\section{Resultados}

La palma Roystonea oleracea (Fig. 1) fue detectada en la región de la costa de Ecuador. La especie es ampliamente cultivada como ornamental en calles y avenidas de la región. Mientras que la naturalización de la especie se observó en Isla Santay, donde la especie sostiene varias poblaciones densas. En esta localidad se colectó la muestra botánica correspondiente (ECUADOR. Guayas: Isla Santay, 18.XII.2015, fr. X Cornejo 8722), la cual fue depositada en el herbario de la Universidad de Guayaquil (GUAY).

\section{Roystonea oleracea (Jacq.) O. F. Cook.}

Tallo solitario, columnar gris hasta $40 \mathrm{~m}$ de alto, 46-66 cm de diámetro. Hojas 15-22 cm, las hojas inferiores sostenidas o más elevadas del plano horizontal, capitel $2 \mathrm{~m}$ de largo; pecíolo 60-100 cm de largo, ráquis 4-4,6 $\mathrm{m}$ de largo; segmentos medios $65-94 \mathrm{~cm}$ de largo y 3-4,9 cm de ancho. Inflorescencia ca. $1 \mathrm{~m}$ de largo y $0,7 \mathrm{~m}$ de ancho; prófilo 46,5-53 cm de largo y 8,8-16 $\mathrm{cm}$ de ancho; bráctea peduncular 1,5 $\mathrm{m}$ de largo, mucho más ancha por encima de la mitad, ápice caudado; raquillas $16-30 \mathrm{~cm}$ de largo y $1,5-2,8$ $\mathrm{mm}$ de diámetro. Flores masculinas blancas; sépalos triangulares, $1,4-1,7 \mathrm{~mm}$ de largo y 1,6-2,6 mm de ancho; pétalos elípticos ovados,
$3-4,8 \mathrm{~mm}$ de largo y $1,4-2,7 \mathrm{~mm}$ de ancho; estambres 6-8 y 4,4-8,8 $\mathrm{mm}$ de largo; filamentos puntiagudos 3-6,9 mm de largo; anteras 3,5-4,7 $\mathrm{mm}$ de largo, ápice recurvado.. Flores pistiladas blancas 2,5-4,5 cm; sépalos reniformes $1,5-1,8$ $\mathrm{mm}$ de largo y 3,3-4,3 $\mathrm{mm}$ de ancho; pétalos ovados, 2,6-3,4 mm de largo; estaminodios 6-lobulado, 1.8-2,5 $\mathrm{mm}$ de largo, libres $0,9-1,2$ $\mathrm{mm}$; gineceo $1.8-2.9 \mathrm{~mm}$ de largo y $1,6-2,5 \mathrm{~mm}$ de diámetro. Frutos elipsoides, gibosos, 12,6-17,6 $\mathrm{mm}$ de largo, 8,2-10,8 $\mathrm{mm}$ de grosor dorsiventral y 7,6-10,4 mm de ancho; epicarpio negro violáceo; endocarpio elipsoide, 9,8-13,7 mm de largo, 6,8 $\mathrm{mm}$ de grosor dorsiventral y $6,8-7,7 \mathrm{~mm}$ de ancho; semilla elipsoide, 7,5-10,5 mm de largo, 4,6-6,1 $\mathrm{mm}$ de grosor dorsiventral y $5,1-6,8 \mathrm{~mm}$ de ancho; rafe circular. Eófilos linear-elípticos, cortos o estipitados, débilmente costados.

En Ecuador, $R$. oleracea suele ser confundida con $R$. regia. Sin embargo, Zone (1996) propone la siguiente combinación de caracteres para distinguir a $R$. oleracea de otras especies de Roystonea: i) segmentos de las hojas arreglados en dos planos a los lados del raquis; ii) hojas de la corona, por lo general, se sostienen en el plano horizontal, y iii) bráctea peduncular cerrada, con el ápice acuminado.

Roystonea oleracea es nativa de las Antillas Menores, Barbados, Trinidad y Tobago, norte de Venezuela y el noreste de Colombia (Kahn \& Moussa 1997; Zona 1996; Pintaud et al. 2008). La especie es un componente de la región de Los Llanos, la cual se extienden desde el pie de la Cordillera Oriental de Colombia hasta el medio y bajo Orinoco en Venezuela. En esta región, $R$. oleracea está presente naturalmente en los bosques de galerías (Pintaud et al. 2008). Los registros globales de la especie disponibles en la base de datos GBIF evidencian que la especie ha sido introducida en Bolivia, Brasil, Costa Rica, Guayana Francesa, Guyana, Honduras, Indonesia, Madagascar, Namibia, Panamá, Puerto Rico y Estados Unidos. En Manaos (Brasil), grabados antiguos que datan de 1865, muestran que $R$. oleracea ya había sido introducida para embellecer vías principales (Khan \& Moussa 1996). Actualmente, la especie parece estar naturalizada en áreas inundables naturales en Brasil, Guayana y Panamá, ocupando áreas pantanosas de la reserva de Uniäo en Brasil (Nascimiento et al. 2013), áreas de ensenadas en Guayana (Henderson 1995) y el bosque de pantano en Panamá (Svenning 2002). 

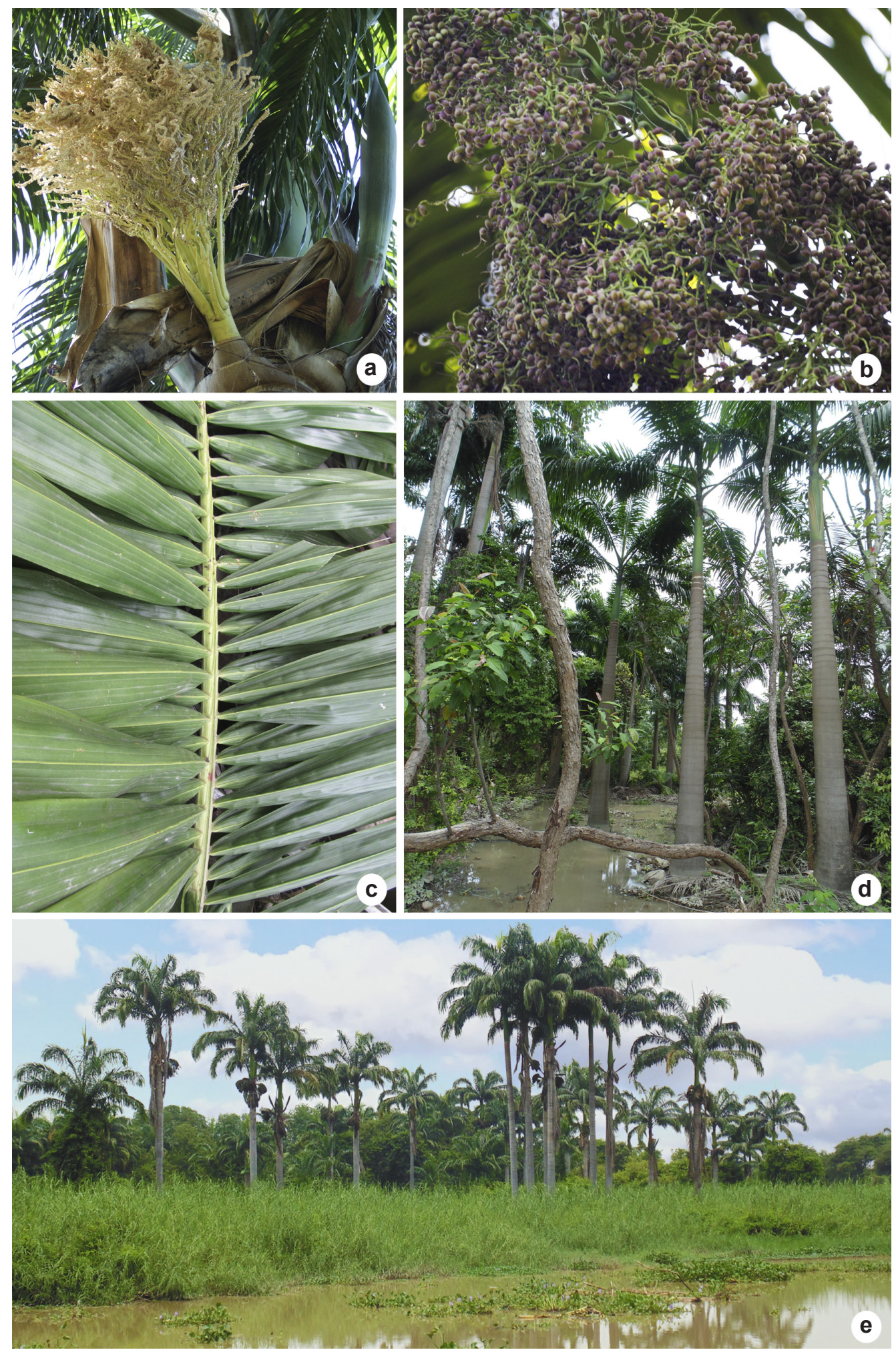

Figura 1 - a. inflorescencia; b. infrutescencia; c. hoja; d-e. individuos adultos de Roystonea oleracea en Isla Santay, ubicada en la región de la costa de Ecuador. Fotos: J.I. Hernández-Rosas, X. Cornejo e I. Herrera.

Figure 1 - a. inflorescence; b. infructescence; c. leaf; d-e. adult individuals of Roystonea oleracea in Isla Santay, located in the region of the coast of Ecuador. Photos: J.I. Hernández-Rosas, X. Cornejo and I. Herrera. 
Roystonea oleracea es una especie dominante en algunos humedales tropicales, específicamente en aquellos caracterizados por presentar una lámina de agua poco profunda $(20-50 \mathrm{~cm})$, bajo nivel de salinidad (2-3\%) y un hidro-periodo que genere fluctuaciones en la profundidad de la lámina de agua (Bonadie 1998). En la isla de Trinidad, la fructificación y germinación de semillas coincide con el inicio de la estación húmeda (Bonadie 1998). Se conoce muy poco sobre la biología reproductiva, dispersión de semillas y repoblación de esta especie de palma. En Venezuela, la germinación de las semillas inicia en la octava semana y el porcentaje de germinación puede variar de $20-50 \%$, siendo este porcentaje mayor (50-80\%) cuando las semillas permanecen en agua por un periodo de 4-6 días (Maciel 2001). Las semillas de las especies del género Roystonea en general son de corta longevidad, menos de un año (Maciel 2001), por tanto es poco probable que sus poblaciones formen un banco de semillas en el suelo. Las semillas de esta palma podrían dispersarse a larga distancia mediante el agua y probablemente por animales. Los frutos de $R$. oleracea son un importante componente de la dieta de especies de loros en Trinidad (Bonadie 1998; Bonadie \& Bacon 2000). En Cuba, se ha reportado que los frutos pueden ser dispersados por murciélagos (Mancina et al. 2007). Mientras que en Brasil, donde ha sido introducida, la dispersión de semillas parece llevarse a cabo por aves y murciélagos (Nascimiento et al. 2013).

\section{Abundancia y cobertura de Roystonea oleracea en Isla Santay}

Se cuantificaron 5.316 individuos adultos de $R$. oleracea (Fig. 2a) los cuales cubren un área aproximada de 43.1 ha $( \pm 14.4 ; n=25)$ en Isla Santay. La mayor densidad de individuos de $R$. oleracea se concentra en cuatro parches de 12 a 40 hectáreas; además, se distinguen individuos y parches pocos densos y aislados (Fig. 2b).

\section{Distribución potencial de}

Roystonea oleracea en Ecuador

El modelo de distribución potencial muestra que en la región de la costa ecuatoriana hay extensas áreas con susceptibilidad climática alta y
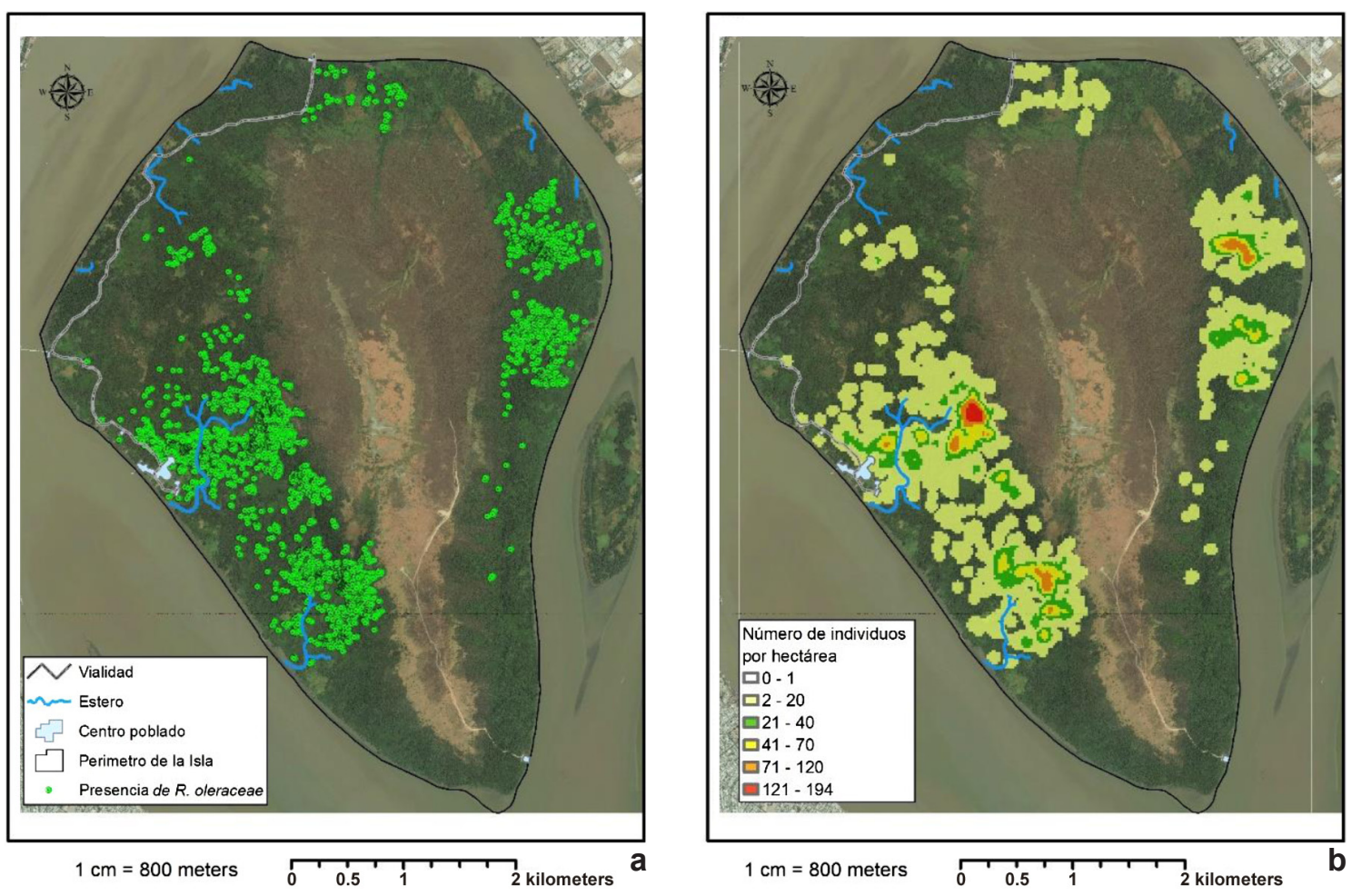

Figura 2 - Roystonea oleracea en Isla Santay, región de la costa, Ecuador - a. ocurrencia; b. densidad. Figure 2 - Roystonea oleracea in Isla Santay, coastal region, Ecuador - a. occurrence; b. density. 
muy alta al establecimiento de R. oleracea (Fig. 3). En esta misma región también hay zonas con media y baja susceptibilidad climática. Se identificaron pequeñas áreas con susceptibilidad climática media y baja al establecimiento de la especie de estudio en la región insular (Islas Galápagos; Fig. 3). En Ecuador continental las áreas susceptibles se superponen con los siguientes humedales Ramsar: i) Reserva Ecológica de Manglares de CayapasMataje, ii) Laguna de Cube, iii) La Segua, iv) Abras de Mantequilla, v) Isla Santay, y vi) Manglares de Churute (Fig. 3). En la Islas Galápagos las zonas predichas como susceptibles al establecimiento de $R$. oleracea no se sobreponen con el único humedal Ramsar presente en estas islas (i.e. Humedales del Sur de Isabela) (Fig. 3).

Las condiciones climáticas bajo las cuales $R$. oleracea podría establecerse incluyen valores de temperatura entre $20-26^{\circ} \mathrm{C}$ promedio anual (Bio 1; Fig. 4a), elevada precipitación entre 1000-3000 $\mathrm{mm}$ al año (Bio 12; Fig. 4b). La variable bio15 y bio2 además indican que estos lugares más susceptibles representan en su mayoría sitios con alta variabilidad en la precipitación y rango de temperatura diario entre los 6 y $10^{\circ} \mathrm{C}$ (Fig. $4 \mathrm{c}-\mathrm{d}$ ).

\section{Discusión}

Nuestro trabajo reporta por primera vez la presencia, y naturalización de Roystonea oleracea en humedales ubicados en la región de la costa de Ecuador. La historia de introducción, las características de la especie y la susceptibilidad climática de la región, podrían propiciar un proceso de invasión por $R$. oleracea en humedales en Ecuador que debe ser monitoreado para evitar impactos negativos en estos ecosistemas.

Como ocurre con otras plantas ornamentales que son introducidas en grandes números, la naturalización de $R$. oleracea en Isla Santay podría haber sido facilitada por la alta presión de propágulos. Aunque poco se conoce sobre la historia de introducción de $R$. oleracea, probablemente la especie fue introducida en los años 70 como planta ornamental por pobladores de la isla, donde la especie encontró condiciones climáticas y ecológicas para su establecimiento

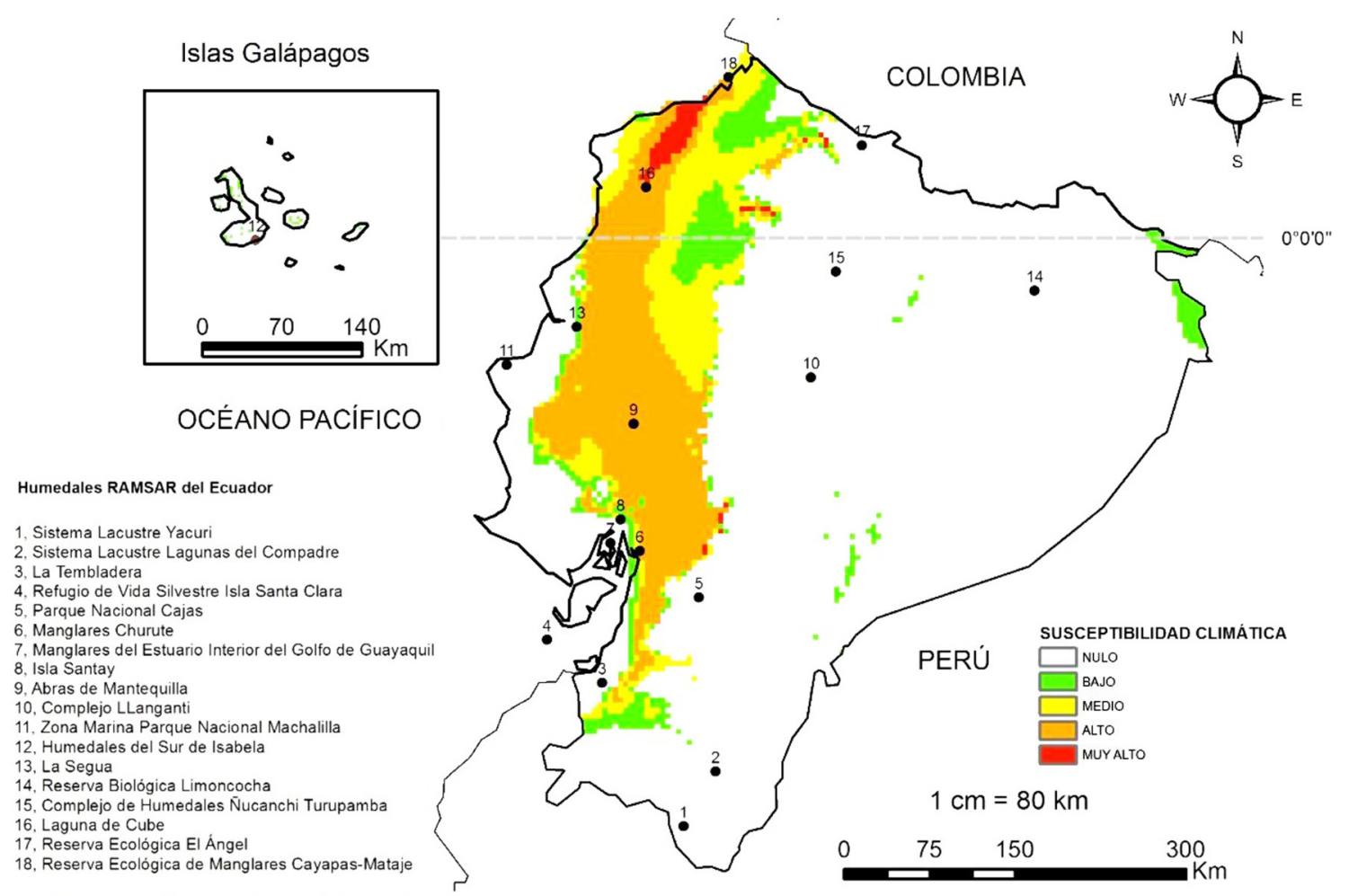

Figura 3 - Susceptibilidad climática al establecimiento de Roystonea oleracea en Ecuador. Se muestran los humedales Ramsar del Ecuador.

Figura 3 - Climatic susceptibility to the establishment of Roystonea oleracea in Ecuador. The Ramsar wetlands of Ecuador are shown. 
exitoso. En Isla Santay, los parches con mayor densidad de $R$. oleracea tienen de 70 hasta 194 individuos adultos por hectárea (Fig. 2b). Esta densidad es similar a la encontrada (152 individuos/ ha) en un área invadida por esta misma especie de palma en un humedal en la Mata Atlántica brasileña (Zuricatto \& Pires 2014).

Una de las primeras evidencias de que una especie tiene el potencial de invadir es que haya sido reportada como invasora en otras regiones similares. $R$. oleracea tiene antecedentes como invasora en otros humedales en el Neotrópico (Henderson et al. 1995; Svenning 2002; Nascimento et al. 2013), lo que quiere decir que la especie tiene las características reproductivas y ecológicas que le permiten invadir este tipo de ambientes. Además,
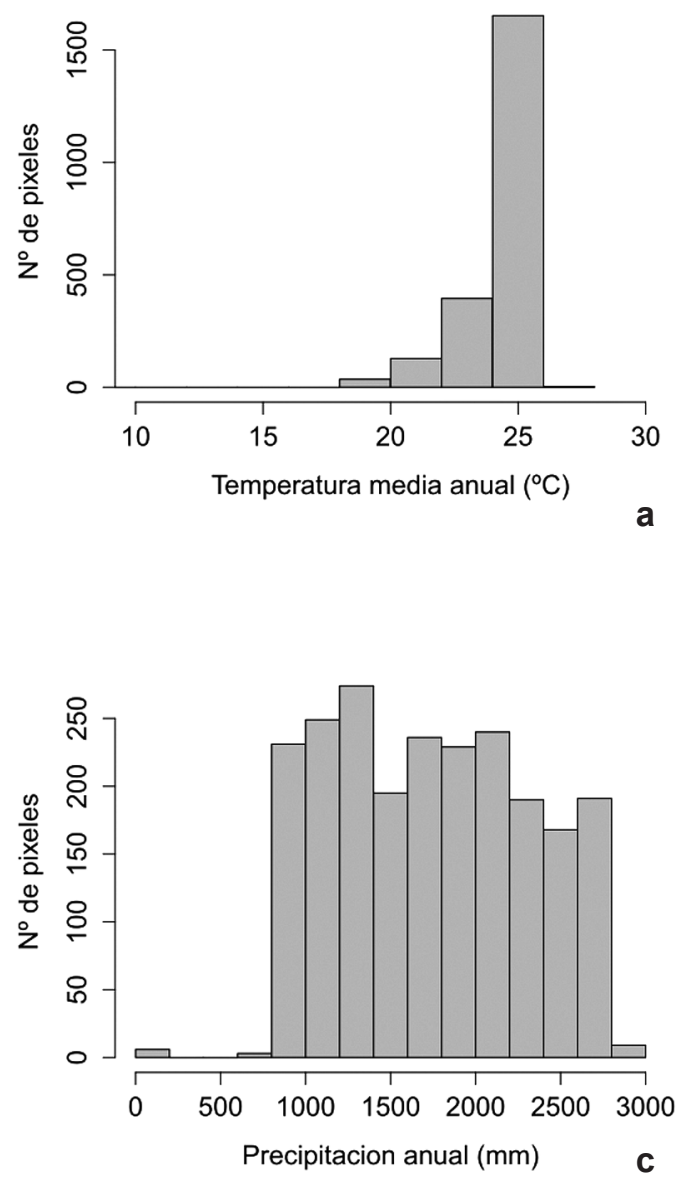

$R$. oleracea tiene algunas características típicas de especies invasoras, tales como alta producción de frutos y la capacidad de dispersar sus semillas a largas distancias (ver Rejmanek \& Richardson 1996; Lloret et al. 2005). Esta última característica puede favorecer su expansión y el establecimiento de nuevos focos de invasión en Isla Santay y otros humedales cercanos.

A escala nacional, el mapa de distribución potencial refleja que la región de la costa de Ecuador es susceptible climáticamente al establecimiento de $R$. oleracea. En esta región hay extensas áreas que presentan condiciones climáticas que fueron predichas por el modelo como adecuadas (zonas naranjas y rojas en la Fig. 3). Sin embargo, esta especie parece ser
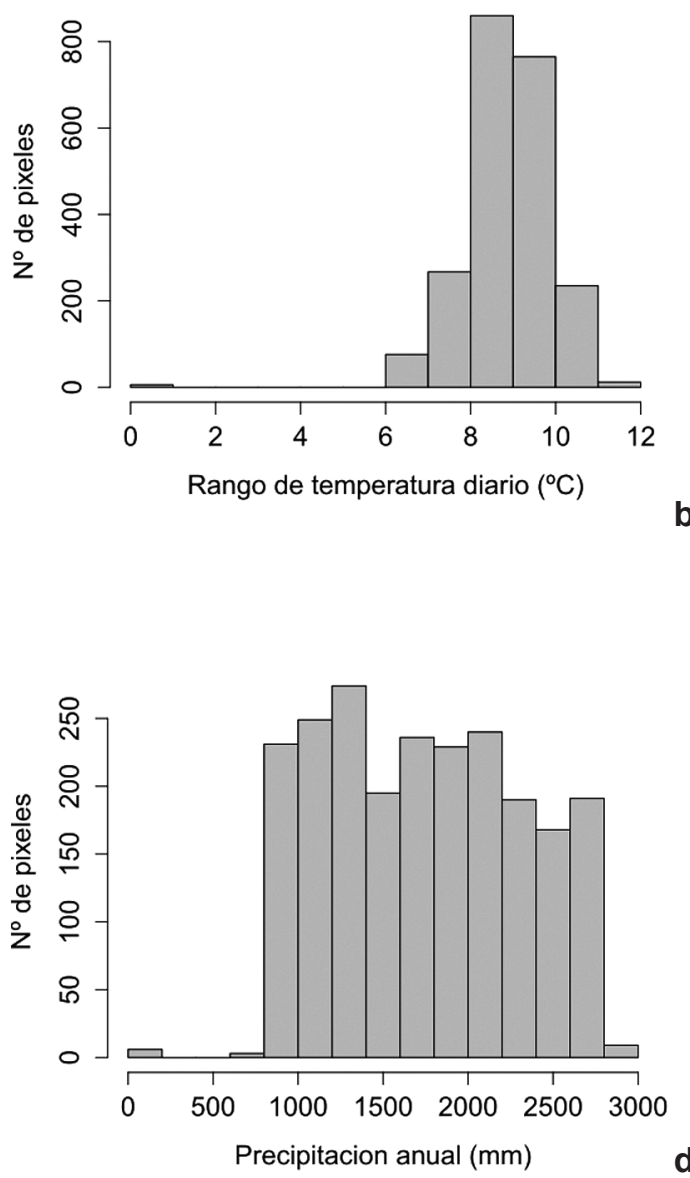

Figura 4 - Distribución de frecuencias de los valores de las variables climáticas en cada píxel predicho por el modelo con alta y muy alta susceptibilidad. Variables climáticas - a. temperatura promedio anual $\left({ }^{\circ} \mathrm{C}\right)$; b. precipitación anual; c. rango de temperatura diario $\left({ }^{\circ} \mathrm{C}\right)$; d. coeficiente de variación en precipitación.

Figure 4 - Frequency distribution of the values of the climatic variables in each pixel predicted by the model with high and very high susceptibility. Climate variables - a. average annual temperature $\left({ }^{\circ} \mathrm{C}\right)$; b. annual precipitation; c. daily temperature range $\left({ }^{\circ} \mathrm{C}\right)$; d. coefficient of variation in precipitation. 
una invasora exclusiva de humedales, por tal motivo las áreas que potencialmente podrían ser invadidas por $R$. oleracea corresponden a las pequeñas zonas con humedales, ubicados dentro de la extensa área identificada como susceptible climáticamente al establecimiento de esta especie. En la región de la costa del Ecuador hay muchas otras zonas de humedales que no tienen categoría Ramsar. Sin embargo, nuestro estudio se restringió a señalar cuáles humedales Ramsar podrían ser afectados por la invasión de $R$. oleracea, debido a que financieramente no es viable monitorear las invasiones en extensas áreas y por tanto es necesario priorizar las áreas que deben ser protegidas. Además de Isla Santay, otros cinco humedales Ramsar, de los 18 que están presentes en Ecuador, son susceptibles climáticamente a la invasión por R. oleracea (Fig. 3). Los humedales de importancia internacional Ramsar son ecosistemas que ofrecen múltiples beneficios socio-ambientales, tales como el mantenimiento de suministro de agua, sostén para la pesca, actividades recreativas, proveer de hábitats únicos para fauna y flora, y en especial para aves acuáticas, entre otros (Ramsar 2006). Dentro de los objetivos de los humedales Ramsar está contribuir con la conservación de la fauna y flora nativa. El cumplimiento de este objetivo puede ser afectado por el establecimiento de una especie introducida, ya que estas especies en general tienden a afectar negativamente a las comunidades de fauna y flora nativa (Vilà et al. 2011). Deben realizarse visitas sistemáticas en los cinco humedales susceptibles a la invasión por $R$. oleraceae, con el fin de evitar que la especie llegue a naturalizarse en estos humedales.

\section{Riesgos asociados a la naturalización} e invasión de Roystonea oleracea

No hay estudios disponibles que evalúen los impactos ocasionados en Ecuador por la invasión de la especie de estudio, sin embargo; en humedales en Brasil se determinó que la invasión por esta palma está asociada a una reducción en la riqueza y abundancia de plántulas de especies nativas (Zucaratto \& Pires 2014). Futuros estudios deberían enfocarse en evaluar los cambios en la composición de especies de plantas nativas asociados a la invasión por $R$. oleracea en Isla Santay y en examinar los efectos de estos posibles cambios en el comportamiento, abundancia y distribución de las aves que hacen uso de este humedal. Por otro lado, ha sido reportado para otras localidades que los frutos de $R$. oleracea son consumidos y dispersados por diferentes especies de aves y mamíferos (Bonadie 1998; Bonadie \& Bacon 2000; Mancina et al. 2007; Nascimiento et al. 2013). La capacidad de una planta exótica de incorporar interacciones mutualistas - para la polinización o dispersión de semillas - con especies de animales nativos es frecuentemente un factor clave para su éxito como especie invasora (Richardson et al. 2000). Para tener una visión más completa del potencial invasivo de $R$. oleracea en Isla Santay y alrededores es necesario determinar si esta especie introducida ha logrado establecer relaciones mutualistas con dispersores potenciales.

Otro riesgo asociado a la invasión de $R$. oleracea es que esta especie podría favorecer la abundancia de triatominos capaces de transmitir el mal de chagas. En Brasil (estado de Goiás) se han encontrado individuos de una especie de chipo (Rhodnius neglectus) asociada a hojas y tallos de $R$. oleracea. Esta especie de triatomino es uno de los vectores del parásito Trypanosoma cruzi, causante del mal de chagas (Vianna et al. 2014). Por tanto se recomienda la realización de estudios que evalúen las poblaciones de triatominos en zonas bajo la influencia de $R$. oleracea y cerca de los centros poblados en Isla Santay.

Recomendaciones para el control de la expansión de Roystonea oleracea

Para contrarrestar la expansión de la especie en Isla Santay recomendamos iniciar la extracción mecánica de individuos (adultos, juveniles y plántulas) en los parches aislados, pequeños y poco densos que fueron identificados en este estudio (Fig. 2). En estos pequeños parches es donde la erradicación es más efectiva y económica (Simberloff 2009). Mientras que los parches grandes y densos deben ser manejados desde la periferia hacia el centro donde la densidad de individuos es mayor, la idea con esto es lograr contener la expansión (Simberloff 2009). Uno de los problemas del control mecánico es que posterior a este, el sustrato queda descubierto de vegetación y se facilita el ingreso de otras especies invasoras, por tanto lo más recomendable es que el control este acompañado de un programa de restauración ecológica (Zavaleta et al. 2001).

El uso de $R$. oleracea como planta ornamental en Ecuador debe evitarse para no favorecer aún más su naturalización e invasión. Particularmente, debe prohibirse en el país el uso de $R$. oleracea como planta ornamental en áreas cercanas a humedales, debido al potencial invasivo de esta especie en 
estos ecosistemas. Además es necesario realizar un monitoreo para confirmar o descartar la presencia de $R$. oleracea en los humedales identificados en este estudio como susceptibles al establecimiento de esta especie exótica, con el fin de prevenir nuevos focos de invasión.

\section{Agradecimientos}

Al proyecto Prometeo de la SENESCYT (Ecuador), que financió una beca de investigación para Ileana Herrera.

\section{Referencias}

Bonadie, W.A. 1998. The ecology of Roystonea oleracea palm swamp forest in the Nariva Swamp (Trinidad). Wetlands 18: 249-255.

Bonadie, W.A. \& Bacon, P.R. 2000. Year-round utilisation of fragmented palm swamp forest by Red-bellied macaws (Aramanilata) and Orange-winged parrots (Amazona amazonica) in the Nariva Swamp (Trinidad). Biological Conservation 95: 1-5.

Brown, B.J.; Mitchell, R.J. \& Graham, S.A. 2002. Competition for pollination between an invasive species (purple loosestrife) and a native congener. Ecology 83: 2328-2336.

Carpenter, G.; Gillison, A. \& Winter, J. 1993. Domail: a flexible modelling procedure for mapping potential distributions of plants and animals. Biodiversity and Conservation 2: 667-680.

D'Antonio, C.M. \& Dudley, T. 1995. Biological invasions as agents of change on island versus mainland. In: Vitousek, P.; Loope, L.L. \& Adsersen, H. (eds.). Islands Biological diversity and ecosystem function. Vol.115. Ecological Studies. Springer-Verlag, Berlin. Pp 103-117.

Delnatte, C. \& Meyer, J.Y. 2012. Plant introduction, naturalization, and invasion in French Guiana (South America). Biological Invasions 14: 915-927.

Dislich, R.; Kisser, N. \& Pivello, V.R. 2002. The invasion of a forest fragment in São Paulo (SP) by the Australian palm Archontophoenix cunninghamiana H. Wendl. \& Drude. Brazilian Journal of Botany 25: 55-64.

Elton, C.S. 2000. The Ecology of invasions by animals and plants: a new foreword by D. Simberloff. The University of Chicago Press, Chicago. 181p.

Groves, R.H. 1998. Recent incursions of weeds to Australia 1971-95. CRC for Weed Management Systems Technical Series 3: 1-74.

Henderson, A.; Galeano, G. \& Bernal, R. 1995. Field guide to the palms of the Americas. Princeton University Press, Princeton. Pp. 421.

Hijmans, R.J.; Cameron, S.E.; Parra, J.L.; Jones, P.G. \& Jarvis, A. 2005. Very high resolution interpolated climate surfaces for global land areas. International Journal of Climatology 25: 1965-1978.
Jørgensen, P.M. \& León-Yánez, S. (eds.). 1999. Catalogue of the vascular plants of Ecuador. Monograps in Systematic Botany from the Missouri Botanical Garden 75: i-viii, 1-1182.

Kahn, F. \& Moussa, F. 1997. El papel de los grupos humanos en la distribución geográfica de algunas palmas en la Amazonía y su periferia. In: Rios, M. \& Borgtoft Pedersen, H. (eds.). Uso y manejo de recursos vegetales: memorias del segundo simposio ecuatoriano de etnobotánica y botánica económica. Abyam Yala, Quito. Pp. 83-99.

Kallimanis, A.S.; Bergmeier, E.; Panitsa, M.; Georghiou, K.; Delipetrou, P. \& Dimopoulos, P. 2010. Biogeographical determinants for total and endemic species richness in a continental archipelago. Biodiversity and Conservation 19: 512-520.

Lonsdale, W.M. 1999. Global patterns of plant invasions and the concept of invasibility. Ecology 80: 15221536.

Lloret, F.; Médail, F.; Brundu, G.; Camarda, I.; Moragues, E.; Rita, J.; Lambdon, P. \& Hulme, P.E. 2005. Species attributes and invasion success by alien plants on Mediterranean island. Journal of Ecology 93: 512520.

Maciel, N. 2001. Emergencia de la palma real venezolana (Roystonea oleracea (Jacq.) O.F. Cook) en función de condiciones variables del fruto y la semilla. Bioagro 13: 105-110.

Maciel, N. \& Mogollón, N. 1995. Variables de la emergencia de semillas germinadas de seis palmas ornamentales. Bioagro 7: 10-16.

Mack, R.N. \& Erneberg, M. 2002. The United States naturalized flora: largely the product of deliberate introductions. Annals of the Missouri Botanical Garden 89: 176-189.

Mack, R.N.; Simberloff, D.; Mark Lonsdale, W.; Evans, H.; Clout, M. \& Bazzaz, F.A. 2000. Biotic invasions: causes, epidemiology, global consequences, and control. Ecological Applications 10: 689-710.

Mancina, C.A.; García-Rivera, L. \& Capote, R.T. 2007. Habitat use by phyllostomid bat assemblages in secondary forests of the 'Sierra del Rosario' Biosphere Reserve Cuba. Acta Chiropterologica 9: 203-218

Meyer, J-Y.; Lavergne, C. \& Hodel, D.R. 2007. Time bombs in gardens: invasive ornamental palms in tropical islands, with emphasis in French Polynesia (Pacific Ocean) and the Mascarenes (Indian Ocean). Palms 52: 71-83.

Nascimento, M.T.; Araújo, R.M.; Dan, M.L.; Netto, E.B.F. \& Braga, J.M.A. 2013. The Imperial Palm (Roystonea oleracea (Jacq.) O.F. Cook) as an invasive species of a wetland in Brazilian Atlantic forest. Wetlands Ecology and Management 21: 367-371.

Navarrete, R. 2008. Ficha informativa de los humedales Ramsar: Isla Santay. Sistema de Información Marino Costera de Ecuador. Ministerio del Ambiente del Ecuador. Disponible en $<\mathrm{http}$ ://simce.ambiente.gob. 
ec/documentos/ficha-informativa-humedales-ramsarisla-santay>. Acceso el 10 noviembre 2015.

Pintaud, J.C.; Galeano, G.; Balslev, H.; Bernal, R.; Borchsenius, F.; Ferreira, E. \& Noblick, L. 2008. Las palmeras de América del Sur: diversidad, distribución e historia evolutiva. Revista Peruana de Biología 15: 7-30.

Ramsar. 2006. Manual de la convención de Ramsar: guía a la convención sobre los humedales (Ramsar, Irán, 1971). 4 ed. Secretaría de la Convención de Ramsar, Gland (Suiza). Disponible en $<$ http://www. ramsar.org/sites/default/files/documents/pdf/lib/ lib_manual2006s.pdf $>$. Acceso el 1 diciembre 2015.

Reichard, S.H. 1997. What traits distinguish invasive plants from noninvasive plants? In: Lovich, J.E.; Randall, J. \& Kelly, M.D. (eds.) Proceedings of the California Exotic Pest Plant Council Symposium, San Diego. Pp. 31-38.

Rejmánek, M. 1996. Species richness and resistance to invasions. In: Orians, D. \& Cushman H. (eds.). Biodiversity and Ecosystem processes in tropical forest. Ecological Studies. Vol. 122. Springer-Verlag, Berlin, Heidelberg. Pp. 153-172.

Rejmánek, M. \& Richardson, D.M. 1996. What attributes make some plant species more invasive? Ecology 77: 1655-1661.

Richardson, D.M.; Allsopp, N.; D'Antonio, C.M.; Milton, S.J. \& Rejmanek, M. 2000. Plant invasions-the role of mutualisms. Biological Reviews 75: 65-93.

Shigesada, N. \& Kawasaki, K. 1997. Biological invasions: theory and practice. Oxford University Press, New York. Pp. 206.
Simberloff, D. 2009. We can eliminate invasions or live with them. Successful management projects. Biological Invasions 11: 149-157.

Svenning, J.C. 2002. Non-native ornamental palms invade a secondary tropical forest in Panama. Palms 46: 81-86.

Vianna, E.N.; Andrade, A.J.D.; Dias, F.B.S. \& Diotaiuti, L. 2014. The exotic palm Roystonea oleracea (Jacq.) O.F. Cook as a rural biotype for Rhodnius neglectus Lent, 1954, in Caçu, state of Goiás. Revista da Sociedade Brasileira de Medicina Tropical 47: 642-645.

Vilà, M.; Espinar, J.L.; Hejda, M.; Hulme, P.E.; Jarošík, V.; Maron, J.L. \& Pyšek, P. 2011. Ecological impacts of invasive alien plants: a meta-analysis of their effects on species, communities and ecosystems. Ecology Letters 14: 702-708.

Wilson, J.R.; Dormontt, E.E.; Prentis, P.J.; Lowe, A.J. \& Richardson, D.M. 2009. Something in the way you move: dispersal pathways affect invasion success. Trends in Ecology and Evolution 24: 136-144.

Zona, S. 1996. Roystonea (Arecaceae: Arecoideae). Flora Neotropica 71: 1-35.

Zavaleta, E.S.; Hobbs, R.J. \& Mooney, H.A. 2001. Viewing invasive species removal in a wholeecosystem context. Trends in Ecology and Evolution 16: 454-459.

Zucaratto, R. \& Pires, A.S. 2014. The exotic palm Roystonea oleracea (Jacq.) O.F. Cook (Arecaceae) on an island within the Atlantic Forest Biome: naturalization and influence on seedling recruitment. Acta Botanica Brasilica 28: 417-421. 